\title{
Development of constitutive equations for hot working of titanium matrix composites
}

\author{
Lihua Du ${ }^{\mathrm{a},{ }^{*}}$, Zhusheng Shi ${ }^{\mathrm{b}}$, Yuanfei Han ${ }^{\mathrm{c}}$, Jie Shao ${ }^{\mathrm{a}}$, Kailun Zheng ${ }^{\mathrm{b}}$, Yong $\mathrm{Li}^{\mathrm{b}}$, Weijie $\mathrm{Lu}^{\mathrm{c}}$ \\ a Department of Metal Forming Technology, AVIC Manufacturing Technology Institute, Beijing, 10024, PR China \\ ${ }^{b}$ Department of Mechanical Engineering, Imperial College London, London SW7 2AZ, UK \\ ${ }^{c}$ Department of Materials Science and Engineering, Shanghai Jiaotong university, Shanghai, 200240, PR China \\ *Idu@imperial.ac.uk
}

\begin{abstract}
This research is devoted to modelling the viscoplastic deformation behaviour and microstructure evolution of particle reinforced titanium matrix composites (TMCs) at hot working conditions. A series of Gleeble hot compression tests were conducted to obtain the stress-strain curves. According to the dominant mechanisms of TMCs during deformation, a set of mechanism-based constitutive equations was developed and fitted based on the experiment data. Lamellar alpha globularisation, dynamic recrystallization and damage were considered and incorporated into the constitutive equations to describe the viscoplastic flow behaviour.
\end{abstract}

\section{Introduction}

To improve the service temperature of titanium alloys further, great importance has been attached to particle reinforced titanium matrix composites (TMCs) ${ }^{[1]}$, which can be fabricated by traditional methods similar to that of titanium alloys. The TMCs exhibit excellent mechanical properties without large increase of fabrication cost ${ }^{[2,3]}$. The formability and hot deformation behaviour of TMCs have been investigated ${ }^{[4,5]}$. Accordingly, the processing window was defined, and the hot working methods, such as isothermal forging, hot extrusion and rolling process have also been employed to improve the microstructure and achieve sheet or bulking materials. ${ }^{[6,7]}$

Near net shape forming methods have great advantages of material utilising rate and excellent materials properties ${ }^{[8]}$. However, few research has been devoted to investigated near net shape forming methods of TMCs. To develop near net shape methods, the optimal processing window under which the materials would have a better formability should be found. Both the geometry accuracy and materials properties of a specific part should be taking into consideration at the same time. Therefore, constitutive equation which can both describe the microstructure evolution and deformation behaviour is essential to understand, simulate and optimise the forming process for TMCs.

Currently, several forms of constitutive equations for TMCs under hot deformation condition have been proposed. Xiao et al. [9] established a power-law based constitutive equation to describe the steady rate creep behaviour of TMCs, and the activation

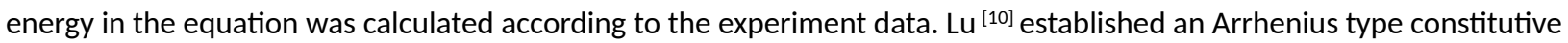
equation to describe the stress-strain curve of Ti matrix composites under hot compression condition. Song ${ }^{[11]}$ modified a Johnson-Cook model to consider the coupled effects of temperature and strain rate.

However, few of present constitutive equations were mechanism-based. This research is dedicated to establishing a set of mechanism-based constitutive equations to describe the deformation behaviour and microstructure evolution at hot deformation condition.

\section{$\underline{2 \text { Material \& Experiment }}$}

\subsection{Material}

The material used in this research, supplied by Shanghai Jiaotong University, was fabricated by in-situ synthesised technology $[2,3]$. The composites mainly consist of ceramic reinforcements with a volume fraction of approximately $2 \%$ (including short whisker TiB and nano scale particle rare earth oxide) and Ti-6Al-4V matrix. The as-received material was isothermal forged in two phase field after casting.

\subsection{Experiment}

A series of high temperature compression tests were conducted on Gleeble Materials Simulator 3800. The samples for hot compression test, with a height of $12 \mathrm{~mm}$ and diameter of $8 \mathrm{~mm}$, were cut down and machined from as-received materials. 
A profile of hot compression test was designed with various temperatures $\left(850^{\circ} \mathrm{C}, 900^{\circ} \mathrm{C}, 950^{\circ} \mathrm{C}\right)$ and strain rates $(0.1 / \mathrm{s}$, $0.01 / \mathrm{s}, 0.001 / \mathrm{s})$. The heating process (i.e. heating rate and soaking time) for every sample is shown in Fig.1.

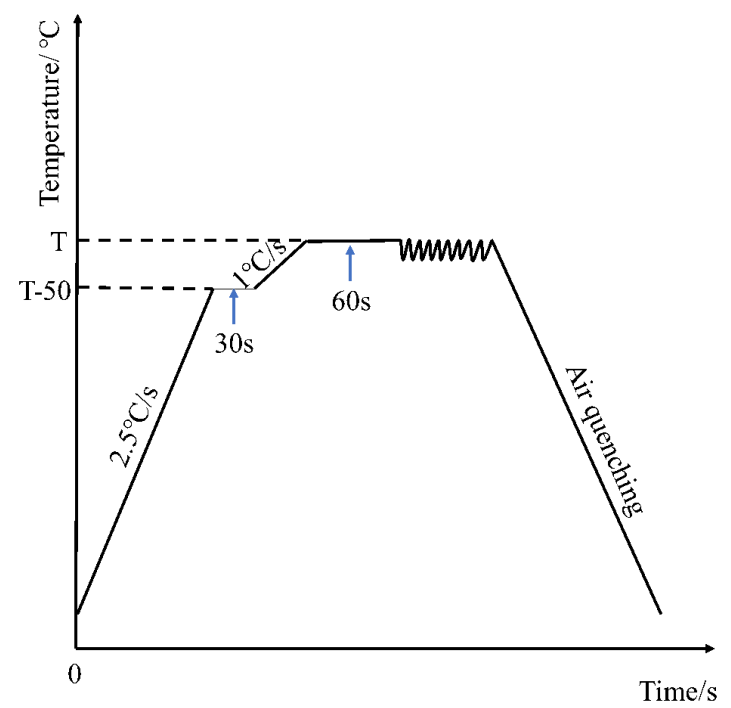

Fig. 1 Thermal process for Gleeble hot compression test specimens

\section{Material Modelling}

This research is devoted to establishing mechanism based constitutive equation of TMCs at hot working condition, under which the material is most likely to be deformed cost-effectively. The constitutive equations are expected to model the viscoplastic deformation behaviour and microstructures that evolve dramatically during deformation.

According to previous research, globularisation of secondary lamellar alpha induced by beta strain ${ }^{[4,5,12]}$, dynamic recrystallization ${ }^{[8]}$, breaking and debonding of ceramic reinforcements ${ }^{[4,5]}$, have been observed and revealed regarding to dual phase titanium matrix composites at hot working condition. There are other mechanisms, such as adiabatic heating ${ }^{[12]}$ and rotation of whisker reinforcements ${ }^{[13]}$. However, based on the dominant deformation mechanism for TMCs at specific deformation conditions that this research has covered, recrystallization and damage are mainly considered for materials modelling in this paper. These mechanisms have significant effects on evolution of grain size, dislocation density and flow stress. Consequently, such effects should be modelled and the relevant variables should be integrated in the constitutive equations.

\subsection{Recrystallization modelling}

Recrystallization is significant process that greatly determines the microstructure and deformation behaviour of titanium alloys and TMCs. The microstructure evolution, i.e. morphology and grain size of matrix alloy have a close connection with recrystallization. The recrystallization in titanium alloys and TMCs mainly takes two forms: globularisation of secondary alpha lath and dynamic recrystallization ${ }^{[4,5,12]}$.

\subsubsection{Globularisation of secondary alpha lath}

Globularisation is considered as a main mechanism that governs the softening phenomenon of dual phase titanium alloys. It occurs due to the localisation of strain into intense shear bands within the alpha lath ${ }^{[12]}$. The extent of globularisation increase with the strain of beta phase.The equation of globularisation has been proposed by Bai et al. ${ }^{[12]}$. The equation takes the formation as follows.

$$
\dot{w}=c_{w}(1-w) \dot{\varepsilon}_{p_{1} \beta}
$$

$w$, which varies from 0 to 1 , represents the extent of globularisation. $c_{\mathrm{w}}$ is a material constant. $\dot{\varepsilon}_{p, \beta}$ is the plastic strain rate of beta phase.

\subsubsection{Dynamic recrystallisation}

The fraction of dynamic recrystallization is represented by $S$ that can be expressed as following equation ${ }^{[14]}$.

$$
\dot{S}=q_{1}(0.1+S)^{q_{2}}(1-S) \bar{\rho}^{2} \frac{d_{0}}{d}
$$


Where $d$ is grain size of primary alpha phase, $d_{0}$ is the initial value of grain size. $q_{1}, q_{2}$ are material constants.

\subsubsection{Effect of recrystallization on dislocation density}

Dislocation accumulates with increasing plastic strain and releases due to softening mechanisms, such as recovery and recrystallization. The normalized dislocation density $\bar{\rho}$, changing from 0 to 1 , can be expressed as follows considering accumulation and reduction of dislocation ${ }^{[15]}$.

$$
\dot{\bar{\rho}}=A(1-\bar{\rho}) \dot{\varepsilon}_{p}^{\prime}-C_{1} \bar{\rho}^{n_{2}}-C_{2} \bar{\rho} \frac{\dot{S}}{1-S}
$$

Where $\varepsilon_{p}$ is plastic strain rate of materials and $A, C_{1}, C_{2}, n_{2}$ are material constants. The first term of the equation governs the accumulation of dislocation density caused by plastic deformation. The second term represents the static recovery of dislocation density, While the third term give the effect of recrystallization on dislocation density.

\subsubsection{Effect of recrystallization on grain size}

In consideration of static grain growth, plastic strain induced grain growth and the strain refinement due to recrystallization. In this case, the grain size of primary alpha is considered mainly. The average grain size of primary alpha, represented by $d$ can be expressed as the equation (4) - (6).

$$
\overline{\rho_{c r}}=q_{3} \bar{\varepsilon}_{p}^{q 4}
$$

Where $\bar{\rho}_{c r}$ means the threshold of dislocation density for recrystallization. $q_{3}, q_{4}$ are material constants. When $\bar{\rho}<\bar{\rho}_{c r}$, the recrystallization would not happen, the grain size can be expressed as follows.

$$
\dot{d}=w_{1}\left(\frac{d}{d_{0}}\right)^{-r_{1}}+w_{2} \dot{\varepsilon}_{p}\left(\frac{d}{d_{0}}\right)^{-r_{2}}
$$

When $\bar{\rho}>\bar{\rho}_{c r}$, the recrystallization would happen and influence the grain size which can be expressed as follows.

$$
\dot{d}=w_{1}\left(\frac{d}{d_{0}}\right)^{-r_{1}}+w_{2} \dot{\varepsilon}_{p}\left(\frac{d}{d_{0}}\right)^{-r_{2}}-w_{3} \dot{S}^{r_{3}}\left(\frac{d}{d_{0}}\right)^{r_{4}}
$$

Where $w_{1}, w_{2}, w_{3}, r_{1}, r_{2}, r_{3}, r_{4}$ are material constants.

\subsection{Damage modelling}

Dislocation density and local stress are likely to intensify around reinforcements because hard reinforcements behave as a barrier to dislocation movements during plastic deformation. When the concentration of local stress reaches a certain extent, debonding between hard reinforcements and ductile matrix, or breaking of reinforcements could occur, which give birth to void and crack successively. Damages of TMCs are quite common to be observed in the specimens after deformation even in the stress state of uniaxial compression test, which is different from titanium alloys. Although the damage seems to not influence the stress-strain curve dramatically in the compression test, still it is important to be predicted and controlled in deformed parts of TMCs.

Based on the continuum damage mechanics ${ }^{[14]}$, a damage factor which represents the fraction of void and crack is given in the form as follows.

$$
\dot{f}_{d}=\eta\left(1-f_{d}\right) \varepsilon_{p}^{\prime}+\frac{D \varepsilon_{p} \sigma \varepsilon_{p}^{\prime}}{1-f_{d}}
$$

Where $D, \eta$ are material constants. The damage is influenced by plastic strain rate, strain and stress.

\subsection{Viscoplastic flow rule}

According to the basic form of power law ${ }^{[12,15,17]}$, the viscoplastic flow can be expressed as following equations when considering the phase composition, grain size evolution, globularisation of secondary alpha lath, dynamic recrystallisation and damage.

$$
\begin{gathered}
\dot{\varepsilon}_{p}=\dot{\varepsilon}_{p, \beta} C_{\beta}(T)+\dot{\varepsilon}_{p, \alpha}\left(1-C_{\beta}(T)\right) \\
\dot{\varepsilon}_{p, \alpha}=\left(\frac{\sigma-R-k_{\alpha}}{K_{\alpha}}\right)^{n_{1}}\left(\frac{d}{d_{0}}\right)^{-u}
\end{gathered}
$$




$$
\begin{gathered}
\dot{\varepsilon}_{p, \beta}=\left(\frac{\sigma-R-k_{\beta}}{K_{\beta}(1-w)}\right)^{n_{1}} \\
\dot{R}=0.5 B \bar{\rho}^{-0.5} \dot{\bar{\rho}} \\
C_{\beta}(T)=\left(\frac{T}{T_{\beta}}\right)^{N}
\end{gathered}
$$

Where $C_{\beta}(T), T_{\beta}, d$ represent beta phase volume fraction, beta phase transformation temperature and grain size of primary alpha respectively. $B, u, n_{1}$ are material constants.

The stress can be calculated by Hooke's law ${ }^{[14]}$.

$$
\sigma=E\left(1-f_{d}\right)\left(\varepsilon_{T}-\varepsilon_{p}\right)
$$

Where $\varepsilon_{T}, \varepsilon_{p}, E$ represent total strain, plastic strain and modulus respectively.

\subsection{Formulation of unified viscoplastic constitutive equations}

According the above discussions, a set of unified viscoplastic constitutive equations consists of equations (1)-(13). The temperature dependent constants can be expressed as follows.

$$
\theta=\theta_{0} \exp \left(\frac{Q_{\theta}}{R T}\right)
$$

Where $\theta$ represents the specific temperature dependent constants. $\theta_{0}$ is coefficient related to $\theta .\left|Q_{\theta}\right|$ is activation energy and $R$ , $T$ are gas constant and absolute temperature respectively.

\section{Results and discussions}

The material constants, which are included in the constitutive equations, are difficult to be determined by analytical method. A numerical method ${ }^{[16]}$ was applied to determine the material constants in the equations based on stress-strain curves obtained by hot compression tests (Table1 and Table 2).

Table 1. Determined temperature dependent material constantsin the unified constitutive equations for TMCs

\begin{tabular}{cccccc}
\hline$K_{\alpha_{0}}(\mathrm{Mpa})$ & $Q_{K_{\alpha}}(\mathrm{J} / \mathrm{mol})$ & $q_{2_{0}}(-)$ & $Q_{q_{2}}(\mathrm{~J} / \mathrm{mol})$ & $c_{w_{0}}(-)$ & $Q_{c_{W}}(\mathrm{~J} / \mathrm{mol})$ \\
\hline 0.28592 & 64870.69 & 64.2 & -31278.84 & 115.1 & -49735.35 \\
$k_{\alpha_{0}}(\mathrm{Mpa})$ & $Q_{k_{\alpha}}(\mathrm{J} / \mathrm{mol})$ & $C_{1_{0}}(-)$ & $Q_{c_{1}}(\mathrm{~J} / \mathrm{mol})$ & $n_{1_{0}}(-)$ & $Q_{n_{1}}(\mathrm{~J} / \mathrm{mol})$ \\
0.66648 & 35465.47 & 565.6 & -51970.74 & 0.010191 & 53648.40 \\
$K_{\beta_{0}}(\mathrm{Mpa})$ & $Q_{K_{\beta}}(\mathrm{J} / \mathrm{mol})$ & $C_{2_{0}}(-)$ & $Q_{C_{2}}(\mathrm{~J} / \mathrm{mol})$ & $B o(-)$ & $Q_{B}(\mathrm{~J} / \mathrm{mol})$ \\
0.13252 & 69187.46 & 0.08966 & 67003.53 & 0.000864 & 116996.31 \\
$k_{\beta_{0}}(\mathrm{Mpa})$ & $Q_{k_{\beta}}(\mathrm{J} / \mathrm{mol})$ & $w_{2_{0}}(-)$ & $\left.Q_{w_{2}} \mathrm{~J} / \mathrm{mol}\right)$ & $w_{3_{0}}(-)$ & $Q_{W_{3}}(\mathrm{~J} / \mathrm{mol})$ \\
0.000102 & 111810.04 & $3.73 \mathrm{E}-14$ & 281958.3 & $3.49 \mathrm{E}+08$ & -121492.2 \\
$E_{0}(-)$ & $Q_{E}(\mathrm{~J} / \mathrm{mol})$ & & & & \\
$1.20 \mathrm{E}+04$ & 5811.18 & & & & \\
\hline
\end{tabular}

Table 2. Determined temperature independent material constants in the unified constitutive equations for TMCs

\begin{tabular}{ccccccc}
\hline$q_{I}(-)$ & $u(-)$ & $A(-)$ & $r_{3}(-)$ & $n_{2}(-)$ & $D(-)$ & $w_{I}(-)$ \\
\hline 150 & 5 & 1.75 & 1.5 & 3.5 & 0.015 & 0.01 \\
$q_{3}(-)$ & $q_{4}(-)$ & $\eta(-)$ & $N(-)$ & $r_{I}(-)$ & $r_{2}(-)$ & $T_{\beta}(\mathrm{K})$ \\
0.002 & 0.045 & 0.015 & 4.1 & 3 & 2 & 1305 \\
\hline
\end{tabular}

Comparison between experiments and modelling results calculated by the unified constitutive equations is shown in Fig. 2 and Fig.3. The stress-strain curves of TMCs exhibit flow softening behaviour in both experiment and modelling. The extent of softening decreases with temperature increasing and strain rate decreasing. A near-steady flow can be seen at the condition of $950^{\circ} \mathrm{C}, 0.001 / \mathrm{s}$, which means the softening reaches the balance with strain hardening. Although it is generally believed the presence of ceramic reinforcements deteriorate the deformability of TMCs, such a behaviour is beneficial to allow the material to stand large plastic deformation without instability and failure. Meanwhile, the discrepancy of flow stress is much larger when the temperature varies from $850^{\circ} \mathrm{C}$ to $900^{\circ} \mathrm{C}$ compared to the case when the temperature varies from $900^{\circ} \mathrm{C}$ to $950^{\circ} \mathrm{C}$. It is assumed that difference of deformation behaviour could be attributed to the transfer of deformation mechanism. The dominant mechanism is lamellar a globularisation that contributes the softening of material, while dynamic recrystallization at lower strain rates and higher temperatures become main mechanism. 


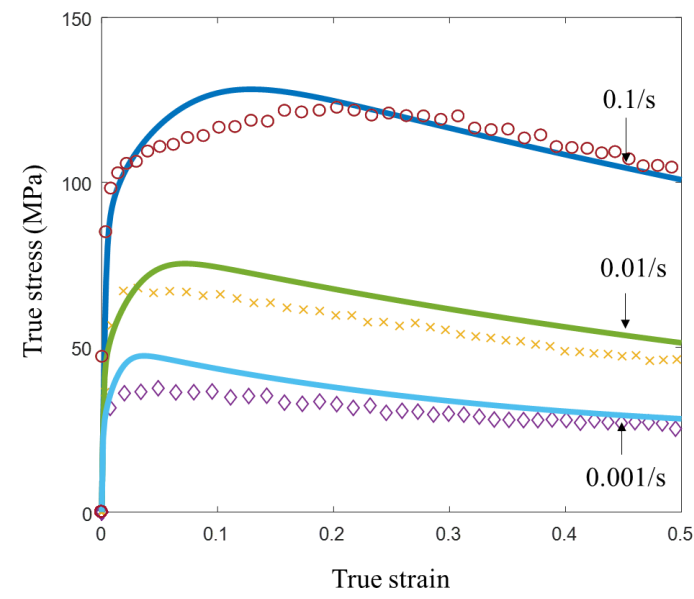

Fig.2 Stress-strain curves at the temperature of $900^{\circ} \mathrm{C}$ and different strain rates(symbols and solid lines represent experiment and modelling respectively)

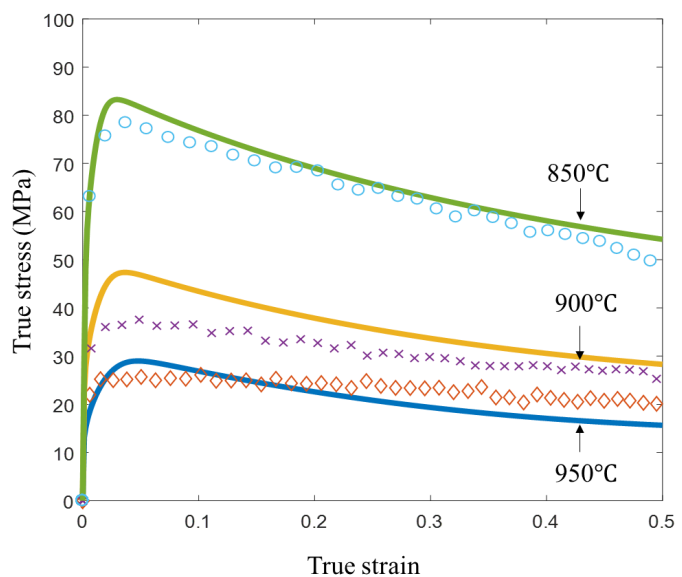

Fig. 3 Stress-strain curves at the strain rate of $0.001 / \mathrm{s}$ and different temperatures(symbols and solid lines represent experiment and modelling respectively)

To verify the constitutive equations further, extra Gleeble hot compression tests at other conditions, which differs from those used to fit the equations, were conducted. The calculated results produced by the constitutive equation and experiments data are shown in Fig.4. The model can predict the general trend of flow stress-strain curves, especially softening phenomenon, and a reasonable agreement of the flow stress value has been obtained.

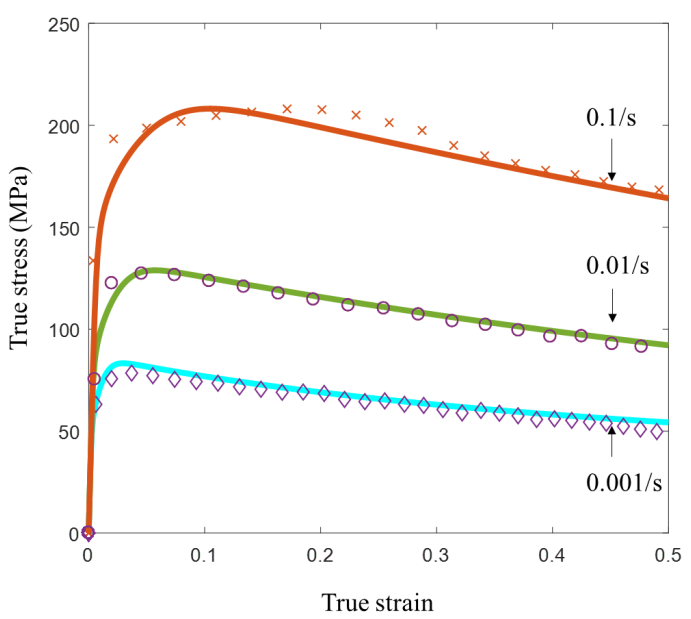

(a) $850^{\circ} \mathrm{C}$ 


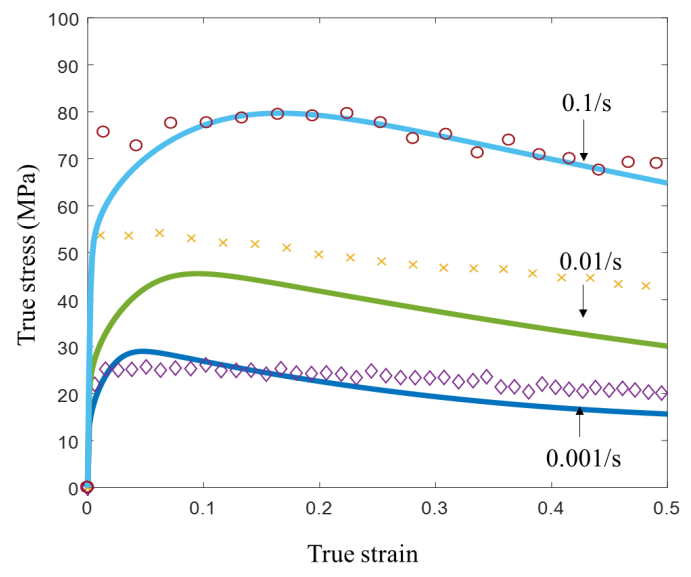

(b) $950^{\circ} \mathrm{C}$

Fig.4 Stress-strain curves for verification from experiments and modelling at different strain rates $(0.1 / \mathrm{s}, 0.01 / \mathrm{s}, 0.001 / \mathrm{s})$ at $850(\mathrm{a})$ and 950 ${ }^{\circ} \mathrm{C}(\mathrm{b})$.

\section{$\underline{5 \text { Conclusion }}$}

The gleeble materials simulation tests reveals that the flow stress of TMCs is sensitive to temperature and strain rate. The stress-strain curves exhibit steady flow softening at the temperature above $900{ }^{\circ} \mathrm{C}$ and strain rate below $10^{-2} / \mathrm{s}$, which indicates the materials have a better capacity to deform at such conditions. Based on the deformation mechanism of TMCs under hot working condition, the lamellar alpha globularisation, dynamic recrystallization and damage were considered and incorporated to the constitutive equations to describe the viscoplastic flow of TMCs. The material constants in the equations were calibrated against the stress-strain curves obtained by hot compression test. The model can predict the general trend of flow stress-strain curves, especially softening phenomenon, and a reasonable agreement of the flow stress value.

\section{Acknowledgement}

The strong support from the Aviation Industry Corporation of China (AVIC) Manufacturing Technology Institute (MTI) for this funded research is much appreciated. The research was performed at the AVIC Centre for Structural Design and Manufacture at Imperial College London. The authors would thank the support of Aviation Science Foundation of China (Grant No: 20173625005).

\section{$\underline{\text { References }}$}

[1] C. Veiga. Mater. Sci. 32 (2012):133-148.

[2] L. Xiao, W. Lu, Jining Qin, Di Zhang. Journal of Materials Research. 23(2008): 3066-3074.

[3] S.C.Tjong, Y. Mai. Composites Science and Technology 68 (2008):583-601.

[4] P. Liu, Y. Hana, P. Qiu. Materials Characterization 146(2018):15-24.

[5] X. Sun, H. Li, Y. Han. Journal of Alloys and Compounds 732(2018): 524-535.

[6] T. Saito. The Journal of The Minerals, 56 (2004): 33-36.

[7] G. Huang, Y. Han, X. Guo. Materials Science and Engineering A 688(2017):155-163.

[8] D. Marini, D. Cunningham, J.R. Corney. Proc. Inst. Mech. Eng. Part B J. Eng. Manuf. 232 (2018): 650-669.

[9] L. Xiao, W. Lu, J. Qin. Materials Science and Engineering A 499 (2009) 500-506.

[10] J. Lu, J. Qin, W. Lu. International Journal of Hydrogen Energy.34 (2009):9266-9273.

[11] W. Song, J. Ning, X. Mao. Materials Science and Engineering A.576(2013):280-289.

[12] Q. Bai. J. Lin, A.Dean, D.S.Balinta, T.Gao, Z.Zhang. Materials Science and Engineering A 559(2013):352-358.

[13] X. Guo, L. Wang, M. Wang, J. Qin, D. Zhang, W. Lu. Acta Materialia 60 (2012) 2656-2667. 
[14] J. Lin, Y. Liu, T. A. Dean. International Journal of DAMAGE MECHANICS, 14(2005): 299-319.

[15] J. Lin, Y. Liu, D. C. J. Farrugia, M. Zhou. Philosophical Magazine, 85(2005):1967-1987.

[16] J. Lin (2015). Fundamentals of materials modelling for metals processing technologies: theories and applications. London: Imperial College Press.

[17] R.C. Picu, A. Majorell. Materials Science and Engineering A326 (2002) 306-316. 